\title{
Scientific Research Review on Dependability of Complex Automotive Systems Developed towards Autonomous Driving
}

\author{
Tímea Lázár-Fülep ${ }^{1 *}$ \\ ${ }^{1}$ Donát Bánki Faculty of Mechanical and Safety Engineering, Óbuda University, Népszínház str. 8, H-1081 Budapest, Hungary \\ * Corresponding author, e-mail: lazar-fulep.timea@edu.bme.hu
}

Received: 26 June 2020, Accepted: 30 June 2020, Published online: 27 January 2022

\begin{abstract}
This brief scientific research review is related to EFOP project titled Dynamics and Control of Autonomous Vehicles meeting the Synergy Demands of Automated Transport Systems (EFOP-3.6.2-16-2017-00016), in which the following scientific consortium is taking part: Széchenyi István University, John von Neumann University, University of Dunaújváros and Óbudai University. The goal of the project is to improve mathematical techniques and processes that assist the increasingly complex design of technical structures and constituents implemented in self-driven automobiles and transport systems, focusing on safety and risk factor maintenance. This means examining the reliability of vehicle sensor networks and developing common risk assessment methods.
\end{abstract}

Keywords

reliability, safety, vehicle system, complex interconnection

\section{Introduction}

Today, one of the most significant social problems is safety, dependability and risk. These have a major impact on engineers and specialists who design and maintain a variety of technical systems on the basis of their area of expertise.

The basic goal of the research is to develop and study the availability of various mathematical answers and well-algorithmic models to support decision making in the field of reliability and safety engineering of automotive systems connected by complex interconnection e.g. sensor networks. In addition to presenting the project, the article also presents the previously written questions.

Further sections of the article: Section 2: Briefly describes the project. Section 3: Represents the problems and approaches mainly discussed. Section 4: Summarizes the general conclusion of the project.

\section{Short description of the scientific research}

The basic goal of this EFOP project titled Dynamics and Control of Autonomous Vehicles meeting the Synergy Demands of Automated Transport Systems is to make research and development circumstances better in terms of human resources and services. In order to ensure appropriate distribution and extensive sponsoring close teamwork with economic community is unavoidable thus it is necessary to be supported and assisted.

Extended consortium aim of shared investigation is having an intensive and trigger role in making economics based on knowledge and improving R\&D prosperity in Hungarian higher education with common power. On the basis of the generated new knowledge bases contribution and teamwork in multinational relations is started. Closer objectives are common work in network and creating scientific teams based on coordinated capacities thus actual individual potentials of the institutions can be multiplied by this synergic effect.

An important aim of the research is that the consortium partners get substantial answers in control and information flow of self-driven vehicles and their systems. The partners have identified seven common research areas arranging them in three core research ways. The conception is established on a combined method and the interface of related scientific issues, with a focus on collaboration on the basis of shared efforts and human resource improvement.

Taking various technical and economic investigations into account the fundamentals below are necessary for self-driven transport through dependable and sustainable operation: 
- On-board surround monitoring tools that work appropriately in many circumstances

- High-precision localization equipment completing sensor data through geographic information in making decisions, which structures, filters, and makes them prepare to sense concerning their spatial relationship

- Communication devices creating data transfer among traffic players, the related infrastructure and objects in surrounding in general

- Observation concepts enabling to investigate conditions of making decisions in reality on the basis of communication and sensor information and assisting control system adjusted to that present state

- Vehicle control algorithms at low-level through complex, locally controlled operation of every vehicle, also actuator process, through making decisions in real time based on detection algorithms in order to safely perform a traffic task given to the vehicle at the desired performance

- Vehicle control at high-level, which allows vehicle groups to be managed along general aspects to achieve traffic management answers in real-time and in optimal conditions

- Use of advanced resources, car propulsion and fuels that are better improved for full automation

- Objectives are determined by the research application and clearly defined in line with the specific project objectives.

\section{Mainly discussed problems and approaches}

Regarding ongoing researches on autonomous driving there are more inevitable issues that must be taken into account, e.g. communication between all participants, algorithms of decision-making, how to enhance trust in appropriate operation.

Today, wireless communication technologies are widespread. This progress has a major impact on the communication of vehicles and on the road side units (RSU) supporting surface transportation. This communication network consists of vehicles and an RSU. Vehicles form an ad hoc network where nodes and network connections change dynamically. Networks consist of fixed nodes and connections. Graph theory is the most effective tool for modeling communication networks. A graph is a finite set of vertices connected by edges; the extract is illustrated in Fig. 1 (Zentai, 2019). Since technical structures consist of network arrangements by mathematical model-based

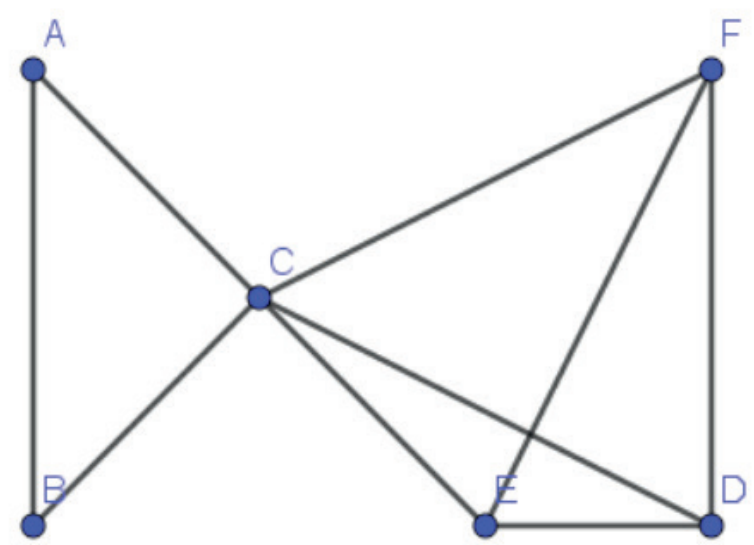

Fig. 1 1-edge-connected and 2-edge-connected graphs

analysis it can be defined if there are relations among elements and subsystems. Concerning a complex system interconnection outline can be a difficult task because of the complicated interconnections. Graph theory as a widespread mathematical solution is about investigating network structured systems with interconnections between elements (Pokorádi, 2018; Pokorádi and Szakács, 2019; Szakács, 2010; 2018; 2019; Szakács and Pokorádi, 2019).

On the one hand, the edge connection models the robustness of the graph against the attack of the connections between the communicating units, and on the other hand, the peak connection models the robustness against the attack of the communicating units. We may have efficient algorithms to calculate the connectivity or edge connection number of a graph, but we cannot perform such a calculation based on the definition of multiple connections. Therefore, we recall the equivalent redefinition of multiple connections (Zentai, 2019).

Systems developed towards autonomous vehicle systems are commonly based on Artificial Intelligence (AI). Time of reaction is lessened by the performance of the AI by processing big amounts of sensor data, and data of circumstances. AI makes decisions much quicker than a driver. Since there is no seamlessly safe system, there will not be $100 \%$ secured system for self-driven cars either. The use of AI for the development of autonomous vehicles is an obvious choice, as decisions need to be processed quickly based on the huge amount of information flowing continuously from various sensors. In the case of industrial AI, where decision-making is based on the analysis of video films, false decisions can result in poor ratings in the rating of flawless or defective products. In the case of human politics, when technical intelligence is used to determine winners, the wrong call can only result in 
gender bias. However, in the case of self-driven vehicles, making the wrong decision can be tantamount to causing accidents and endangering people's lives. In the following some situations are presented when decision-making process of AI can be manipulated. The ability to communicate with other automobiles can solve a number of problems, such as pot holes. An additional benefit for vehicles would be the ability to disseminate their information to make each other ready for traffic or irregular road traffic, or to avoid dangerous situations. The problem is, how differences can be shared on map and in reality? Is it needed to be sent only to the center (or for road operation if necessary) or to the surrounding area? In the second option, the load on the control center is reduced, not all information need to be disseminated to everyone, so there would be less network traffic. In this case, there is a need for a built-in device that can transfer and receive data under a standardized protocol soon, and with controls that separate the data from the recipient (center or neighborhood). It is suggested that before transferring information from diverse places for making decisions, the vehicle incorporates an artificial unit based on intelligence (External Manipulation Recognition System, EMRS) improved by the manufacturers, focusing on the manufacturer's will to consider hazards. Further decisions are needed on which unit is liable for identifying the threat of manipulation from outside and giving suggestions for appropriate assessment of information from various places for making decisions, identifying and analyzing the condition (Kiss, 2019; Péter et al., 2020).

For those systems with the request of advanced reliability concerning their safety-criticality, sensitivity analysis is an adequate process to estimate the sensitivity of the reliability model if there are modifications in the input parameters like component reliability. If minor changes in reliability of the elements show relatively big alterations in the reliability of the system, the structure is supposed to be parameter sensitive. Modifications can be recent variations expected later, indeterminate modifications, or supposed modifications that need to be considered as part of the design. Sensitivity analyzes are generally understood in terms of how concluding remarks are derived from a calculation or evaluation depend on input data, and also focus on further empirical documents in which efforts could be made to improve input estimates that would result in the most improvements in output estimation. Sensitivity measurements are useful, according to many scientists, as a test on model authenticity, or to answer questions like this: the variables in the model are reasonably connected. The adaptability of linear mathematical diagnostic modeling methodology has been shown for creating Linear Fault Tree Sensitivity Model (LFTSM), Linear Sensitivity Model of System Reliability (LSMoSR). These are segmental method tools with matrix-algebraic process on the basis of linearized mathematical diagnostic procedure of gas turbine and jet engines (Pokorádi and Seebauer, 2019).

Another sensitive issue is the significant development of human-robot interaction, or often so-called "human-machine interaction" (HMI), in recent years, which is a direct consequence of ever-evolving detection algorithms and actuators. It is structured to identify key points for action as a guideline for the research community to enhance progress. The security of such interactions has been a much debated topic in the industrial and research communities, addressing this issue in human-robot collaboration in the same field of work. The development of autonomous systems is an iterative process, facilitating technological and regulatory advancement. The biggest breakthrough in recent years in this area has been the emergence of AI-based solutions in safety-critical systems, causing significant uncertainty in terms of operational security. This allows manufacturers to place products on the market with functions that are not fully understood and controlled, which put users at risk. Due to the fact that today's autonomous service robots require a high level of collaboration with the human operator, proper training and feature description are essential before commissioning the system, both in autonomous control and in other areas of human-operated robotics (Takács et al., 2019).

The following notion of Situation Awareness (SA) is applied for describing how the human drive can observe and understand the situation. It is a dangerous case if the driver loses SA. Getting back SA during handover is essential regarding safety issues, because SA is necessary for the driver to find an answer to the problem rising when a handover is under progress. Therefore, creating systems which assist drivers getting back SA is vital in handover management. The manner the driver handles the autonomous structure and reacts to a situation of handover is thought as an issue of Human-Automation Interaction (HAI). Trust in Automation (TiA) is a critical element of HAI systems, since TiA influences human decision leading to interaction. In general, TiA is typically classified by two areas: compliance and reliance. The benefit of applying reliance and compliance is that they can be measured by observable behavior (Drexler et al., 2019). 
In fact, many road accidents occur due to human error or negligence. Fully automated vehicles can reduce the frequency and severity of vehicle collisions. The development of safety protocols for self-driven passenger cars is a highly researched area, focusing on immediate decisions based on mileage sensor data. However, the causes of many accidents cannot be addressed with this model (careless other drivers and pedestrians, road slippage, etc.), but can be well calculated from existing accident databases. A new method is proposed based on experience in the field of road safety. Using data mining algorithms to locate potential hot spots and analyzing those using statistical methods, a list of dangerous areas of the road network is obtained. This catalog can be very useful for an autonomous vehicle to take appropriate precautionary measures for preventing unexpected accidents (Kertész et al., 2019).

\section{References}

Drexler, D. A., Takács, Á., Nagy, T. D., Haidegger, T. (2019) "Handover Process of Autonomous Vehicles - Technology and Application Challenges", Acta Polytechnica Hungarica, 16(9), pp. 235-255. https://doi.org/10.12700/APH.16.9.2019.9.13

Kertész, G., Felde, I., Szénási, S., Nádai, L. (2019) "Road Accident Black Spot Analysis to Support Autonomous Vehicle Control", In: IEEE International Conference and Workshop in Óbuda on Electrical and Power Engineering (IEEE CANDO-EPE 2019), Budapest, Hungary, pp. 225-228.

https://doi.org/10.1109/CANDO-EPE47959.2019.9110973

Kiss, G. (2019) "External manipulation recognition modul in selfdriving vehicles", In: IEEE 17th International Symposium on Intelligent Systems and Informatics (SISY 2019), Subotica, Serbia, pp. 231-234.

https://doi.org/10.1109/SISY47553.2019.9111547

Péter, T., Szauter, F., Rózsás, Z., Lakatos I. (2020) "Integrated application of network traffic and intelligent driver models in the test laboratory analysis of autonomous vehicles and electric vehicles", International Journal of Heavy Vehicle Systems, 27(1/2), pp. $227-245$.

https://doi.org/10.1504/IJHVS.2020.104422

Pokorádi, L. (2018) "Graph model-based analysis of technical systems", IOP Conference Series: Materials Science and Engineering, 393, Article number: 012007. https://doi.org/10.1088/1757-899X/393/1/012007

Pokorádi, L., Seebauer, M. (2019) "Sensitivity Analysis of Bridge Structure Systems' Reliability", In: IEEE 13th International Symposium on Applied Computational Intelligence and Informatics (SACI 2019), Timisoara, Romania, pp. 370-375. https://doi.org/10.1109/SACI46893.2019.9111556

\section{Conclusion}

Concerning the introduced and summarized problems and approaches, without being exhaustive, this inter- and transdisciplinary research area still includes many open issues and challenges. The consortium-based cooperation provides an effective scientific research work and a result oriented outlook for the future.

\section{Acknowledgement}

The research presented in this paper was carried out as part of the EFOP-3.6.2-16-2017-00016 project in the framework of the New Széchenyi Plan. The completion of this project is funded by the European Union and co-financed by the European Social Fund.

Pokorádi, L., Szakács, T. (2019) "Graph Modelling of Pneumatic Vehicle Control System", In: Proceedings of the 3th Agria Conference on Innovative Pneumatic Vehicles - ACIPV 2019, Eger, Hungary, pp. 43-51.

Szakács, T., Pokorádi, L. (2019) "Pneumobil vezérlési rendszerének gráfvizsgálata" (Graph analysis of pneumobil control system), Repüléstudományi Közlemények, 31(1), pp. 53-68. (in Hungarian) https://doi.org/10.32560/rk.2019.1.5

Szakács, T. (2010) "Modellierung und Simulation des Zugwinkels zwischen Anhänger und Zugmaschine" (Modelling and simulation of tow angle between agricultural tractors and trailers), Landtechnik, 65(3), pp. 178-181. (in German) https://doi.org/10.15150/1t.2010.480

Szakács, T. (2019) "Modelling and Validation of a Pneumobil", In: Proceedings of the $3^{\text {th }}$ Agria Conference on Innovative Pneumatic Vehicles - ACIPV 2019, Eger, Hungary, pp. 31-35.

Szakács, T. (2018) "Pneumatic modelling of a pneumobil", In: Proceedings of the 2nd Agria Conference on Innovative Pneumatic Vehicles ACIPV 2018, Eger, Hungary, pp. 25-30.

Takács, Á., Rudas, I. J., Haidegger, T (2019) "Computational-Level Framework for Autonomous Systems: a Practical Approach", In: IEEE 23rd International Conference on Intelligent Engineering Systems (INES 2019), Gödöllö, Hungary, pp. 87-94. https://doi.org/10.1109/INES46365.2019.9109522

Zentai, D. (2019) "Graph Theoretic Risk Classification in Transportation Networks", In: IEEE 17th International Symposium on Intelligent Systems and Informatics (SISY 2019), Subotica, Serbia, pp. 227-230.

https://doi.org/10.1109/SISY47553.2019.9111480 